\title{
Investigation of the Electron Transfer between Levodopa and Zinc Porphyrins at Bionic Interface
}

\author{
Yanru Fan ${ }^{1}$, Bingxu Feng ${ }^{1}$, Yu Huang ${ }^{1, *}$, Xiaoquan $\mathrm{Lu}^{2, *}$ \\ ${ }^{1}$ Key Laboratory of Hui Ethnic Medicine Modernization, Ministry of Education, Ningxia Engineering \\ and Technology Research Center of Hui Medicine Modernization, College of Pharmacy, Ningxia \\ Medical University, Yinchuan 750004, China \\ ${ }^{2}$ Key Laboratory of Bioelectrochemistry \& Environmental Analysis of Gansu Province, College of \\ Chemistry \& Chemical Engineering, Northwest Normal University, Lanzhou 730070, China \\ *E-mail: huangyunxmu@ 163.com, taaluxq@gmail.com
}

doi: $10.10964 / 2018.04 .27$

Received: 4 December 2017 / Accepted: 6 February 2018 / Published: 6 March 2018

\begin{abstract}
In this paper, the interface between two immiscible electrolyte solutions (ITIES) is adopted as the bionic interface model to investigate the interfacial electron transfer (ET) process of levodopa by using thin-layer cyclic voltammetry. Three zinc porphyrins with a systematic variation in structure are used as the reactants in the organic phase for the supply of varied overall driving forces at the ITIES. The consecutive two-step ET reactions between levodopa and three zinc porphyrins with the corresponding ET rate constants are evaluated precisely. The relationship between the interfacial ET rate constants and the overall driving force are also studied. It is found that the ET kinetics of the bimolecular reactions at the ITIES obeys the Marcus theory in a wide potential region. The results of this work will assist in our understanding of the interfacial electron-transfer process of levodopa in vivo.
\end{abstract}

Keywords: levodopa; electron transfer; porphyrin; thin-layer cyclic voltammetry

\section{FULL TEXT}

(C) 2018 The Authors. Published by ESG (www.electrochemsci.org). This article is an open access article distributed under the terms and conditions of the Creative Commons Attribution license (http://creativecommons.org/licenses/by/4.0/). 